\title{
Toward forward genetic screens in malaria-causing parasites using the piggyBac transposon
}

\author{
Brendan S Crabb ${ }^{1,2,3 *}$, Tania F de Koning-Ward ${ }^{4}$ and Paul R Gilson ${ }^{1,3}$ \\ See research article: http://www.biomedcentral.com/1471-2164/12/155
}

\begin{abstract}
The ability to analyze gene function in malariacausing Plasmodium parasites has received a boost with a recent paper in $B M C$ Genomics that describes a genome-wide mutagenesis system in the rodent malaria species Plasmodium berghei using the transposon piggyBac. This advance holds promise for identifying and validating new targets for intervention against malaria. But further improvements are still needed for the full power of genome-wide molecular genetic screens to be utilized in this organism.
\end{abstract}

The development of tools for the genetic manipulation of the causative agents of malaria - protozoan parasites of the genus Plasmodium - has been arduous and frustratingly slow. The drive to obtain such tools is motivated by the ongoing public-health significance of this disease malaria remains a global health and economic catastrophe for which new drugs and an effective vaccine are desperately needed, and if these are to be rationally designed, new insights into Plasmodium biology, particularly in understanding the function of individual gene products, are in turn required. An illustration of this is provided by the new vaccine candidates that are arising from studies identifying specific parasite antigens as essential or important for red blood cell invasion. Manipulation of the genes encoding these candidates was key to dissecting the relative importance and the precise nature of their biological roles [1]. However, progress in this and related endeavors has been slow, because genetic manipulation of Plasmodium parasites remains laborious. Indeed two issues in particular have dogged efforts to develop a array of robust tools to analyze gene function

*Correspondence: crabb@burnet.edu.au

'Macfarlane Burnet Institute for Medical Research and Public Health, Melbourne, Victoria 3004, Australia

Full list of author information is available at the end of the article in Plasmodium: (1) low transformation efficiency whereby very few parasites in a population receive DNA during an individual transfection experiment, and (2) an inability to use RNA interference approaches (which is an alternative to traditional transformation methods) because the specific RNAi machinery is lacking in this organism [2].

Nevertheless, steady progress is being made and a range of molecular genetic approaches to dissect the function of the 5,300 or so Plasmodium genes - including transgenesis, gene knockout, site-directed mutagenesis and knockdown of gene expression - are now available and have been used to good effect [3]. Such tools have been developed both in Plasmodium falciparum, the main cause of human malaria, and in the rodent model system Plasmodium berghei. In fact the development of tools for investigating $P$. berghei has often outpaced that for $P$. falciparum, largely because of the greater efficiency with which plasmid DNA can be introduced in this organism. Apart from P. berghei's greater tractability to genetic manipulation, this model has much to offer because the entire life cycle - through blood, insect-borne and liver stages - can be readily completed in an experimental setting. This is much more difficult to achieve in any non-rodent system. Because many genes are shared across Plasmodium species and are considered orthologous, dissection of gene function in P. berghei can be hugely informative to human malaria biology.

Two major obstacles remain in the development of molecular genetic tools for Plasmodium species: the lack of a simple but robust system to conditionally ablate the function of genes essential to the blood-stage cycle and the lack of a highly efficient random mutagenesis system to allow classical forward genetic screens to recover mutants with interesting phenotypes. A paper by Fonager et al. [4] in BMC Genomics has made great strides in addressing the second of these obstacles.

\section{What's new?}

Fonager and colleagues [4] have developed a system that introduces the piggyBac transposon into the genome of $P$. 


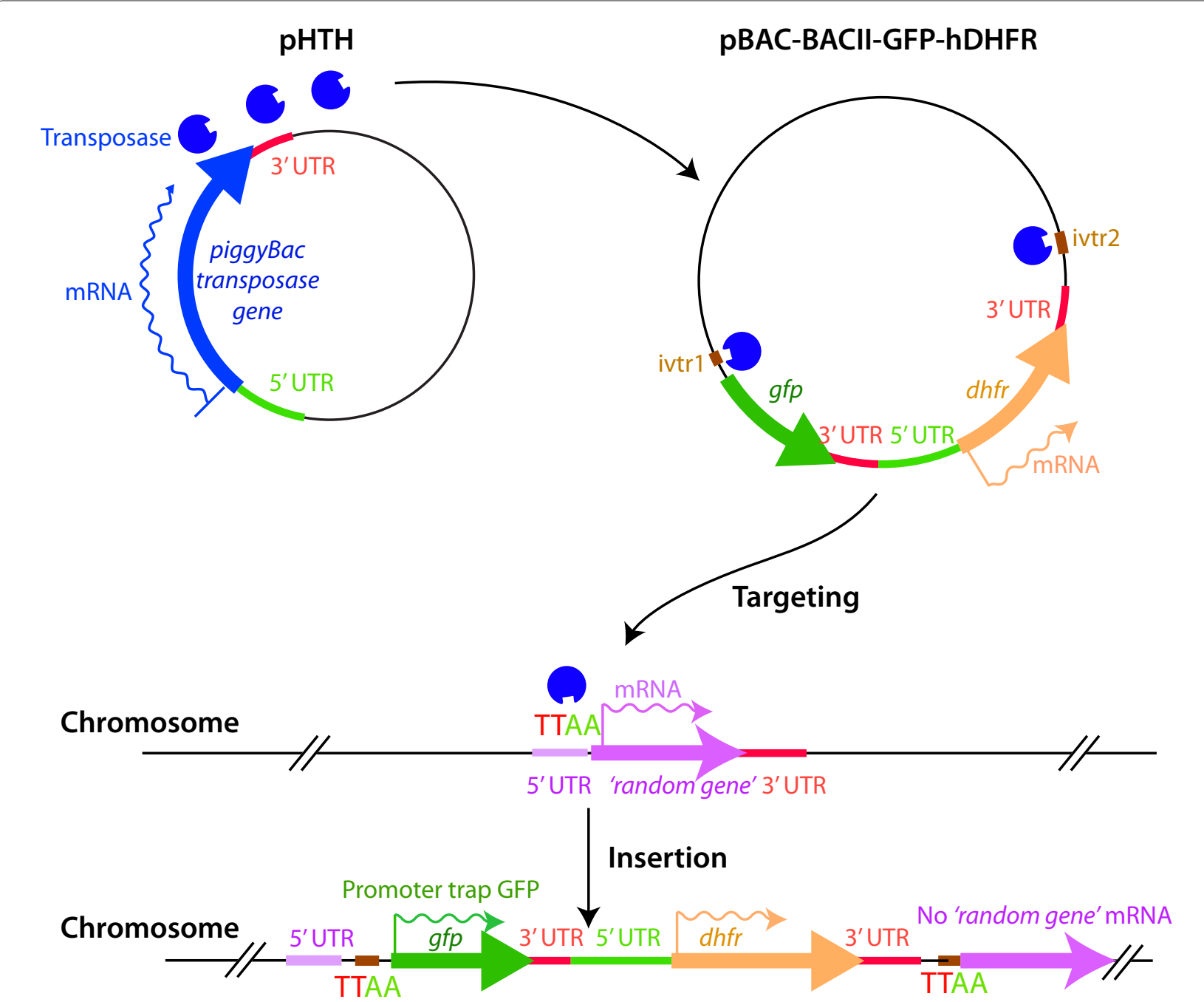

Figure 1. Use of the piggyBac transposon system to disrupt gene function and 'trap' promoter elements. The piggyBac transposase encoded by the pHTH plasmid can integrate a gene cassette (carried on the pBAC-BACII-GFP-hDHFR plasmid) flanked by piggyBac-specific inverted repeats into random TTAA sites within the P. berghei genome [4]. The gene cassette contains a promoter-less green fluorescent protein (gfp) gene and a human dihydrofolate reductase ( $h d h f r$ ) selectable marker. The function of $P$. berghei genes can be ablated by disrupting their coding sequences or their promoters by insertion of the gfp-hdhfr cassette. If the cassette inserts downstream of a gene promoter (as shown here) this promoter can drive expression of $g f p$, which provides information about the timing of expression of the disrupted gene.

berghei. Originally recognized in virus-infected insect cells, piggyBac has been developed as a tool for insertion mutagenesis in a wide range of systems, including mammalian cells. DNA elements flanked by piggyBac sequences can insert into TTAA sequences throughout any genome, as long as the cells also express the piggyBac transposase, the enzyme required for piggyBac transposition (Figure 1).

Fonager et al. [4] build on earlier successes in the Adams laboratory using the same transposon to mutate P. falciparum $[5,6]$. What is new in the current study, apart from the fact that the system has been modified for $P$. berghei, is the markedly improved efficiency of insertion, some 16-18 times that seen in P. falciparum. In the
P. berghei system, 50 or more insertion events can be achieved in a single transfection. Moreover, integration was encouragingly random and included frequent integration into coding sequences, which was not commonly seen in P. falciparum. This approach can therefore be used to disrupt gene expression (see Figure 1), and the authors demonstrate targeting by piggyBac of genes for proteins non-essential to blood-stage growth [4].

Another advance described is the stable integration of the transposase gene into the genome of $P$. berghei. The consequent continuous expression of the transposase allows excision of an integrated piggyBac element and its reinsertion at another genomic location, thus increasing the potential coverage of transposon insertion resulting 
from a single transfection experiment [4]. Together, these features mean that screening for genes responsible for particular phenotypes is now more feasible and practicable in Plasmodium parasites. Demonstrating the potential for such 'forward genetic' screens, Fonager et al. [4] show that it is possible to identify transcriptional promoters in the $P$. berghei genome by 'trapping' a promoter-less green fluorescent protein marker gene flanked by piggyBac elements downstream of a functional promoter (Figure 1).

\section{Where to now?}

So what is the potential of the piggyBac mutagenesis system for genome-wide screens in P. berghei? For example, will it be possible to identify at a genome-wide level all the genes essential, or dispensable, for bloodstage growth? To date, several medium-scale geneknockout approaches have been published, obtaining 20-80 gene-targeting events per study $[7,8]$. These programs were enormously labor intensive, however, and fall far short of the genome-wide knockouts needed.

Another question to be asked of any new technique is can it identify the gene(s) responsible for a measurable phenotype, the genetic equivalent of finding a needle in haystack. Such forward genetic screens are particularly needed for Plasmodium parasites, in which nearly $50 \%$ of Plasmodium genes have no recognizable orthologs outside the genus, as no previous knowledge about gene function is required [9]. In forward genetic screens in model organisms, genomes are generally randomly mutated by radiation, chemical mutagens or insertional mutagenesis using transposons, after which mutants are identified by phenotypic assay and the phenotype tied back to the gene that has been mutated. Such approaches require mutagenesis at a 'saturation' level where every gene is perturbed in a single experiment. While some progress has been made in achieving insertional mutagenesis in Plasmodium, the systems developed to date to not approach the 'saturation' levels required.

It is probably fair to say that even with the improved efficiency of piggyBac insertion demonstrated by Fonager et al. [4], this system still falls short of a 'saturating' mutagenesis procedure that could be easily used for screens. The authors estimate that up to 50 transfection experiments would need to be performed to achieve this level of integration. Most promise for the future probably lies in further improvements to the re-mobilization approach demonstrated by Fonager et al., in which the transposase remains expressed in the parasite cell and facilitates the transposition of piggyBac from one insertion site to another. More frequent and controllable remobilization is probably required to make such an approach effective in achieving frequent insertion events. Perhaps this could be achieved by higher-level expression of the transposase and by controlling expression of the transposase with a conditionally regulatable system. Several methods for doing this are now emerging.

Because parasites are grown and transfected in the haploid blood-stages, the piggyBac insertion approach will, unfortunately, only be useful for the functional analysis of genes that are not essential to blood-stage survival, that is, it will not be possible to recover mutants in which piggyBac has inserted into genes that have a role in blood-stage development. The very essentiality of these genes is of particular interest, as they are likely to be the best targets for future drugs and vaccines. To investigate essential genes will require combining the piggyBac system with one of the conditional expression systems currently available. One possibility would be to place the anhydrotetracycline (ATc)-regulated geneexpression system within the piggyBac inverted terminal repeats [3], allowing the piggyBac-flanked ATc-regulated promoter to jump into and replace the promoter of a random gene. The ATc-regulated promoter would be in the switched-on state, in case the downstream random gene is essential. Once parasites containing these inserted promoters have been cloned, the addition of ATc would switch off expression of the targeted gene, producing a rapid and observable phenotype if the gene is essential.

In summary, this work in P. berghei [4] builds on the less efficient piggyBac mutagenesis system developed for P. falciparum and provides a clear path for saturating genome-wide mutagenesis to be achieved in this organism. Such approaches are needed if the huge amount of genomic information now available is to be dissected and exploited to produce new tools for malaria control. This need is as urgent as ever, given the continued lack of a licensed malaria vaccine and concern over the emergence of parasite strains tolerant to artemisinin derivatives [10].

\section{Acknowledgements}

Our work is supported by the National Health and Medical Research Council (NHMRC) of Australia and the Victorian Government Operational Infrastructure Support Program.

\section{Author details}

1 Macfarlane Burnet Institute for Medical Research and Public Health, Melbourne, Victoria 3004, Australia. ${ }^{2}$ The University of Melbourne, Melbourne, Victoria 3010, Australia. ${ }^{3}$ Monash University, Clayton, Victoria 3800, Australia. ${ }^{4}$ Deakin University, Waurn Ponds, Victoria 3217, Australia.

Published: 31 March 2011

\section{References}

1. Cowman AF, Crabb BS: Invasion of red blood cells by malaria parasites. Cell 2006, 124:755-766.

2. Baum J, Papenfuss AT, Mair GR, Janse CJ, Vlachou D, Waters AP, Cowman AF, Crabb BS, de Koning-Ward TF: Molecular genetics and comparative genomics reveal RNAi is not functional in malaria parasites. Nucleic Acids Res 2009, 37:3788-3798.

3. Meissner M, Breinich MS, Gilson PR, Crabb BS: Molecular genetic tools in Toxoplasma and Plasmodium: achievements and future needs. Curr Opin Microbiol 2007, 10:349-356. 
4. Fonager J, Franke-Fayard BMD, Adams JH, Ramesar J, Klop O, Khan MS, Janse CJ, Waters AP: Development of the piggyBac transposable system for Plasmodium berghei and its application for random mutagenesis in malaria parasites. BMC Genomics 2011, 12:155.

5. Balu B, Chauhan C, Maher SP, Shoue DA, Kissinger JC, Fraser MJ Jr, Adams JH: piggyBac is an effective tool for functional analysis of the Plasmodium falciparum genome. BMC Microbiol 2009, 9:83.

6. Balu B, Shoue DA, Fraser MJ Jr, Adams JH: High-efficiency transformation of Plasmodium falciparum by the lepidopteran transposable element piggyBac. Proc Natl Acad Sci USA 2005, 102:16391-16396.

7. Maier AG, Rug M, O'Neill MT, Brown M, Chakravorty S, Szestak T, Chesson J, Wu Y, Hughes K, Coppel RL, Newbold C, Beeson JG, Craig A, Crabb BS, Cowman AF: Exported proteins required for virulence and rigidity of Plasmodium falciparum-infected human erythrocytes. Cell 2008, 134:48-61.

8. Tewari R, Straschil U, Bateman A, Bohme U, Cherevach I, Gong P, Pain A, Billker $\mathrm{O}$ : The systematic functional analysis of Plasmodium protein kinases identifies essential regulators of mosquito transmission. Cell Host Microbe 2010, 8:377-387.

9. Gardner MJ, Hall N, Fung E, White O, Berriman M, Hyman RW, Carlton JM, Pain A, Nelson KE, Bowman S, Paulsen IT, James K, Eisen JA, Rutherford K, Salzberg SL, Craig A, Kyes S, Chan MS, Nene V, Shallom SJ, Suh B, Peterson J, Angiuoli S, Pertea M, Allen J, Selengut J, Haft D, Mather MW, Vaidya AB, Martin DM, et al:: Genome sequence of the human malaria parasite Plasmodium falciparum. Nature 2002, 419:498-511.

10. Yeung S, Socheat D, Moorthy VS, Mills AJ: Artemisinin resistance on the Thai-Cambodian border. Lancet 2009, 374:1418-1419.

doi:10.1186/1741-7007-9-2

Cite this article as: Crabb BS, et al:: Toward forward genetic screens in malaria-causing parasites using the piggyBac transposon. BMC Biology 2011, $9: 21$ 